\title{
The Impact of Weather on Influenza-like IIIness Rates in Chicago
}

\author{
Shital Shah 1 , Dino Rumoro1, Gordon Trenholme ${ }^{1}$, Gillian Gibbs ${ }^{* 1}$, Marilyn Hallock ${ }^{1}$ and \\ Michael J. Waddell ${ }^{2}$
}

${ }^{1}$ Rush University Medical Center, Chicago, IL, USA; ${ }^{2}$ Pangaea Information Technologies, Chicago, IL, USA

\section{Objective}

To develop a statistical model to account for weather variation in influenza-like illness (ILI) surveillance.

\section{Introduction}

Weather events such as a heat wave or a cold snap can cause a change to the number of patients and types of symptoms seen at a healthcare facility. Understanding the impact of weather patterns on ILI surveillance may be useful for early detection and trend analysis. In addition, weather patterns limit our ability to extrapolate data collected in one region to a different region, which may not share the same weather or periodic trends. By modeling these sources of variation, we can factor out their effects and increase the sensitivity of our overall surveillance system.

\section{Methods}

The time frame for the retrospective study was 2 years (training set - January 1, 2010 through December 31, 2010 and testing set January 1, 2011 through December 31, 2011). The data included in the study were: daily reading of high and low temperature $\left({ }^{\circ} \mathrm{F}\right)$, wind direction, wind gust direction, wind speed (mph), wind gust speed (mph), precipitation (inches) and wind chill $\left({ }^{\circ} \mathrm{F}\right)$.

The daily and seven day moving average (MA) ILI rates for the emergency department (ED) were calculated using the GUARDIAN (Geographic Utilization of Artificial Intelligence in Real-Time for Disease Identification and Alert Notification) surveillance system. In addition, the 7 day MA ILI rate was categorized into Widespread (ILI $\geq 12.5 \%$ ), Imminent $(12.5 \%<$ ILI $\geq 10 \%)$, and Normal (ILI rate $<10 \%)$ levels. We included the day of the week, weekend/weekday status, and holiday status (the six national holidays +/- two days) to account for variations in ED utilization due to patient preferences.

Finally, for all of the weather related data we included a one day lag as well as 3 day MA to smooth out variations. Descriptive and correlation analyses were conducted. Thereafter, we applied both linear regression (dependent variable $=7$ day MA ILI rate) as well as data mining algorithm (decision rules with dependent variable $=$ 7 day MA ILI rate categories). The generated models were validated with the testing dataset.

\section{Results}

Prior day weather data related to high temperature $(\mathrm{r}=-0.76)$, low temperature $(\mathrm{r}=-0.76)$, wind gust speed $(\mathrm{r}=-0.11)$ and wind chill $(\mathrm{r}=-$ $0.76)$ were negatively correlated with the 7 day MA ILI rate. For the 3 day MA weather data, high temperature $(\mathrm{r}=-0.78)$, low temperature $(\mathrm{r}=-0.77)$, wind chill $(\mathrm{r}=-0.78)$, wind gust direction $(\mathrm{r}=-0.17)$, wind gust speed $(\mathrm{r}=-0.18)$, and precipitation $(\mathrm{r}=-0.12)$ were negatively correlated with the 7 day MA ILI rate. The plot of 3 day MA low temperature and 7 day MA ILI rate is presented in Figure 1.

Based on regression analysis, the main significant variables were 3 day MAs for low temperature $(\beta=-0.6 \%)$, wind chill $(\beta=0.43 \%)$, wind speed $(\beta=0.14 \%)$, high temperature $(\beta=-0.05 \%)$, wind gust speed $(\beta=-0.02 \%)$, and wind direction $(\beta=-0.01 \%)$. Some of the sample significant rules based on a decision rules algorithm were as follows:
- IF 7 day MA high temperature > 47.96 AND Yesterday's high temperature > 46.04 AND 7 day MA wind chill > 48.31 THEN 7 day MA ILI rate $=$ Normal $($ confidence $=99.7 \%)$

- IF 7 day MA high temperature $\leq 43.34$ AND holidays $=$ NO AND 7 day MA wind direction $\leq 260$ AND 7 day MA wind speed $>13.35$ EN 7 day MA ILI rate $=$ Imminent $($ confidence $=87.5 \%)$

- IF 7 day MA high temperature $\leq 43.34$ AND 7 day MA low temperature $\leq 7.04$ THEN 7 day MA ILI rate $=$ Widespread (confidence $=86 \%$ )

The accuracy of detecting the ILI category was $76 \%$ and $67.4 \%$ for training and testing datasets, respectively.

\section{Conclusions}

We evaluated multiple weather related parameters for determining ILI rates using statistical and data mining approaches. The significant variables affecting ILI rates consisted of 3 day MA related weather data (i.e., low temperature and wind chill). Thus, these factors should be used in determining ILI rates and associated clinical and operational preparations. Other factors such as day of the week and holiday status had limited contributions in determining ILI rates.

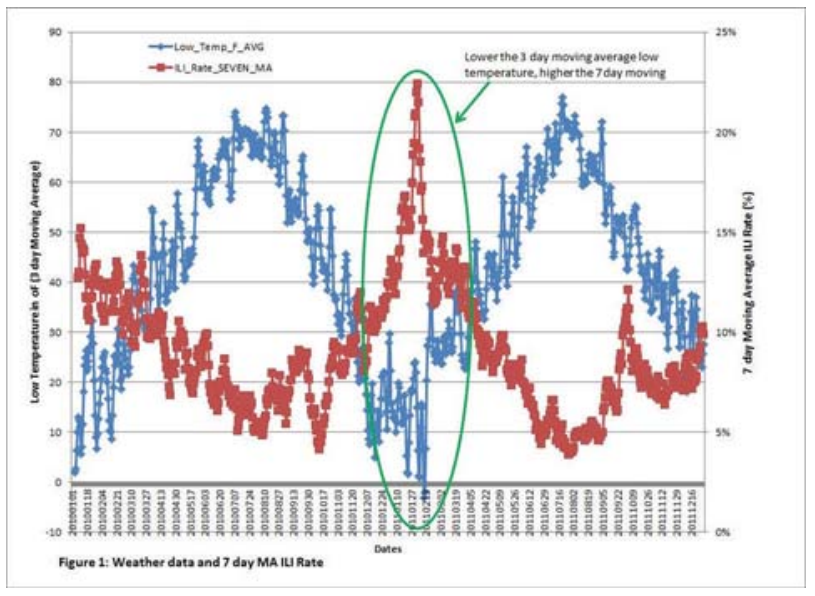

\section{Keywords}

Influenza-like illness surveillance; GUARDIAN; weather patterns

\section{Acknowledgments}

GUARDIAN is funded by the US Department of Defense, Telemedicine and Advanced Technology Research Center, Award numbers W81XWH-09-1-0662 and W81XWH-11-1-0711.

\section{*Gillian Gibbs}

E-mail: Gillian_Gibbs@rush.edu 